\title{
Termination Analysis of CHR revisited
}

\author{
Paolo Pilozzi ${ }^{\star}$ and Danny De Schreye \\ Dept. of Computer Science, K.U.Leuven, Belgium \\ paolo.pilozzi@cs.kuleuven.be danny.deschreye@cs.kuleuven.be
}

\begin{abstract}
Today, there exist two distinct direct approaches to prove termination of CHR programs. Both are applicable on separate classes of CHR programs. One approach, by T. Frühwirth, proves termination of CHR programs without propagation rules. A second approach deals with CHR programs with propagation rules. Due to its extended scope to such programs, it fails to prove termination for a class of CHR programs without propagation that are in the scope of Frühwirth's approach. In this paper, we introduce a termination condition for CHR which is strictly more powerful than both of these and deals with a new class of programs. Keywords: Constraint Handling Rules, Analysis, Termination.
\end{abstract}

\section{Introduction}

Constraint Handling Rules (CHR), created by Thom Frühwirth [10], is a declarative programming languages. It is a concurrent, committed-choice, logic programming language. CHR is constraint-based and has guarded rules that rewrite multisets of atomic formulas. Its simple syntax and semantics make it well-suited for implementing arbitrary solvers $[10,13]$. It is the latter feature of the language that caused its success and impact on the research community $[8,14,15]$.

Many solvers were implemented in CHR, some examples can be found in $[3,12]$. However, to make the language appealing to the larger public, thorough analysis techniques need to be developed to improve both comprehension of and compilation schemes for the language. One of the most important properties of CHR programs is termination. As the execution of multi-headed rules is fairly complicated, mistakes are easily made, leading to unwanted infinite computations. Also, other important properties of CHR programs, such as confluence $[2,9]$, require that a constraint solver is terminating.

Termination analysis of CHR programs received increasing attention in recent years. The main work in the field was presented some years ago in [11]. There, Frühwirth demonstrates that termination proof methods from Logic Programming (LP) and Term-Rewrite Systems (TRS) are applicable to the CHR context. By measuring the multiset size of the constraint store and formulating conditions on simplification rules, he was able to successfully prove an important class of CHR programs terminating. Unfortunately, propagation rules cause explicit increases. Since such rules only add constraints, a different approach was required for programs with propagation rules.

\footnotetext{
* Supported by I.W.T. Flanders - Belgium
} 
Recently, a second direct approach was presented in [16]. Beside programs with simplification, it proves termination of programs with propagation. This was possible by considering a fire-once policy for propagation rules $[1,8]$. A new termination condition was formulated. The condition compares individual constraints in rules, as such guaranteeing finite addition of constraints when executing a CHR program. However, in the case of a simplification only programs, it is less powerful than the approach for CHR without propagation.

In this paper, we present a new approach to prove termination of general CHR programs. Our approach generalizes both of the existing direct approaches and is able to prove a new class of CHR programs terminating. We develop for this purpose a new measure for CHR states, based on a new concept: the propagation store. The latter represents constraints which can be added to a state by only propagating on that state. By also introducing the notion of a token store, to prevent trivial non-termination by propagation rules, we prove decreases on any kind of rule by composing all three stores into a single lexicographical description of a CHR state.

Overview. In a preliminary section, we recall CHR by its syntax and theoretical operational semantics. There, we introduce the concept of a propagation store. The next section discusses termination of CHR programs. First, we introduce some general notions, adapted from termination analysis in other declarative languages. Then, we recall the existing approaches, to be able to later compare them with our approach. Finally, we present our approach, where we first discuss a ranking condition on propagation and afterwards one on simplification. We prove that our conditions are sufficient to prove termination. In Section 4, we discuss our method. Finally, in Section 5, we draw conclusions.

\section{CHR syntax and semantics}

In this section, we recall syntax and semantics of CHR $[1,8,10,13]$ and introduce new concepts w.r.t. semantics, used for termination of CHR programs.

\subsection{Syntax of CHR}

CHR manipulates conjunctions of constraints.

Definition 1 (Constraint). A constraint is syntactically defined as a special first-order predicate $c\left(t_{1}, \ldots, t_{n}\right)$ of arity $n \geq 0$. We distinguish built-in constraints from CHR constraints. Built-in constraints are pre-defined and solved by an underlying constraint solver CT. CHR constraints are user-defined and solved by a CHR program $P$.

A CHR program relates conjunctions of constraints by three different kinds of rules. A simplification rule replaces constraints by simpler constraints, a simp-

agation rule replaces constraints conditional on the presence of other constraints and a propagation rule adds constraints without removing any. 
Definition 2 (CHR program). Let $H_{k}, H_{r}$ and $C$ denote conjunctions of CHR constraints and let $G$ and $B$ denote conjunctions of built-in constraints. A CHR program $P$ is a finite set of CHR rules of the following form:

Simplification rule: Propagation rule: Simpagation rule: $H_{r} \Leftrightarrow G\left|B, C . \quad H_{k} \Rightarrow G\right| B, C . \quad H_{k} \backslash H_{r} \Leftrightarrow G \mid B, C$.

CHR rules are named by adding "rulename @" in front of the rule.

The next example program in CHR computes prime numbers.

Example 1 (Primes). The primes example consists of two different kinds of rules:

test@ $\operatorname{primes}(M) \backslash \operatorname{primes}(N) \Leftrightarrow N>M, N \bmod M$ is $0 \mid$ true.

generate@ $\operatorname{primes}(N) \Rightarrow N>2 \mid N p$ is $N-1, \operatorname{primes}(N p)$.

The first rule, a simpagation rule, tests whether a generated prime number has a divisor. If this is the case, it is removed. The second rule, a propagation rule, generates numbers for prime evaluation, top-down.

In the following, we refer by simplification to both simplification and simpagation, as these have a similar behavior w.r.t. termination.

\subsection{The theoretical operational semantics of CHR}

A CHR program defines a state transition system, where the transition relation is given by the rules in the program and by the underlying CT for solving builtins. Declaratively, simplification defines a logical equivalence between removed constraints $H_{r}$ and added constraints $B \wedge C$, provided that the kept constraints $H_{k}$ are present in the constraint store and that the guard $G$ holds: $G \rightarrow\left(H_{k} \rightarrow\right.$ $\left.H_{r} \leftrightarrow B \wedge C\right)$. For propagation rules, there are no removed constraints. Added constraints are therefore a consequence of constraints present in the constraint store: $G \rightarrow(H \rightarrow B \wedge C)$.

Operationally, rules are applied exhaustively on the CHR constraints in the constraint store, until an answer state is reached. Rule application is nondeterministic, meaning that we can choose to fire any of the rules applicable to a CHR state. This choice is however a committed choice, it cannot be undone. When solving built-ins, this results either in a failed state or in an introduction of bindings for variables, called a computed answer substitution (c.a.s.).

Constraint store. The constraint store is a collection of labeled CHR constraints and built-in constraints. Simplification replaces constraints with "simpler" ones. Propagation only adds constraints to complete state information.

Definition 3 (Constraint store). The constraint store is a set $S$ of uniquely labeled CHR constraints $c \sharp i$ and built-in constraints $b$. We define $\operatorname{chr}(c \sharp i)=c$ to obtain the constraint and $i d(c \sharp i)=i$ to obtain the label. 
Labeling constraints is required to prevent trivial non-termination. This kind of non-termination is caused by propagation in the program. Propagation rules do not remove constraints and therefore are infinitely applicable on the same combination of constraints. In order to keep track of which combinations of constraints that have caused which propagation rules to fire, a fire-once policy is introduced on combinations of labeled constraints.

Token store. As propagation rules need an applicability condition to avoid trivial non-termination $[1,10]$, we introduce the token store. This store keeps information about how propagation rules can be applied on a given set of CHR constraints. Once a propagation rule has been applied to these constraints, the appropriate token is removed, so that the rule cannot be reapplied on the same combination of constraints, as such implementing a fire-once policy.

Definition 4 (Token store). Let $P$ be a CHR program and $S$ a constraint store. Then a token store $T$ given $S$, is a set of tokens $\left(R_{i}, i d_{1}, \ldots, i d_{n}\right)$, where $\left(R_{i} @ h_{1}, \ldots, h_{n} \Rightarrow G \mid B, C\right)$ is a propagation rule in $P$ and where $c_{j} \sharp i d_{j}$ are constraints in $S$, such that $\exists \sigma \theta: C T=c_{1}=h_{1} \sigma \wedge \cdots \wedge c_{n}=h_{n} \sigma \wedge G \sigma \theta$. Here, $\sigma$ is a match substitution for the heads and $\theta$ a c.a.s. for guard satisfaction.

Our definition of the token store is a more strict version of the one in [1]. There, constraints have to be unifiable with heads. In such a setting, tokens can appear in the token store, not corresponding to applicable rules. It covers however for propagation rules that become applicable as a consequence of solving built-ins. We disregard such programs. Therefore in our case, tokens are also only added as a consequence of adding CHR constraints.

Definition 5 (Addition of tokens). Let $P$ be a CHR program, $S$ a constraint store and $C_{0}$ a labeled $C H R$ constraint added to the constraint store $S$, then

$$
\begin{aligned}
T_{\left(C_{0}, S\right)}^{A}=\left\{\left(R, i_{1}, \ldots, i_{n}\right) \mid\right. & \left(R @ h_{1}, \ldots, h_{n} \Rightarrow G \mid B, C\right) \in P, \text { where } \\
& \left\{c_{1} \sharp i_{1}, \ldots, c_{n} \sharp i_{n}\right\} \subseteq C_{0} \cup S \text { such that } \\
& C_{0} \in\left\{c_{1} \sharp i_{1}, \ldots, c_{n} \sharp i_{n}\right\} \text { and } \\
& C T \models \exists \sigma \theta:\left(c_{1}=h_{1} \sigma\right) \wedge \cdots \wedge\left(c_{n}=h_{n} \sigma\right) \wedge G \sigma \theta
\end{aligned}
$$

If multiple constraints $C_{0}=\left\{C^{1}, \ldots, C^{n}\right\}=\left\{c^{1} \sharp i^{1}, \ldots, c^{n} \sharp i^{n}\right\}$ are added, then

$$
T_{\left(C_{0}, S\right)}^{A}=T_{\left(C^{1}, S\right)}^{A} \cup T_{\left(C^{2}, C^{1} \cup S\right)}^{A} \cup \cdots \cup T_{\left(C^{n}, C^{1} \cup \cdots \cup C^{n-1} \cup S\right)}^{A}
$$

Note that $\sigma$ is a match substitution and $\theta$ a c.a.s.

When removing constraints from the store, the invalid tokens are removed.

Definition 6 (Elimination of tokens). Let $T$ be a token store and $C_{0}=$ $\left\{C^{1}, \ldots, C^{n}\right\}$ labeled $C H R$ constraints removed from the constraint store $S$, then $T_{\left(C_{0}, T\right)}^{E}=\left\{\left(R, i_{1}, \ldots, i_{n}\right) \in T \mid \exists C^{j} \in C_{0}: i d\left(C^{j}\right) \in\left\{i_{1}, \ldots, i_{n}\right\}\right\}$

Simplification corresponds to $T^{\prime}=\left(T \backslash T_{\left(H_{r}, T\right)}^{E}\right) \cup T_{\left(C, S \backslash H_{r}\right)}^{A}$, where $H_{r}$ are the CHR constraints removed by simplification and $C$ the CHR constraints added. Propagation on the other hand corresponds to $T^{\prime}=\left(T \backslash\left\{\left(R, i_{1}, \ldots, i_{n}\right)\right\}\right) \cup T_{(C, S)}^{A}$, where $\left\{\left(R, i_{1}, \ldots, i_{n}\right)\right\}$ is the token removed when applying the propagation rule. 
CHR state and CHR transition relation. A CHR state is a tuple of two elements: the constraint store and the token store. It is annotated with a fresh integer value, used to label constraints which enter the constraint store.

Definition 7 (CHR state). A CHR state is a tuple $\langle S, T\rangle_{\nu}$, where $S$ is the constraint store and $T$ the token store. Every state is annotated with a fresh integer $\nu$, not yet assigned to a constraint. An initial state is a tuple $\left\langle S, T_{(S, \emptyset)}^{A}\right\rangle_{v}$, with $v$ a fresh integer value. In a final state no more transitions are possible.

Transitions between states occur in two different settings. Either at least one constraint is removed and we simplify the state or no constraints are removed and we propagate on the state. The transition relation $\rightarrow$ between $\mathrm{CHR}$ states, given $C T$ for built-ins and $P$ for CHR constraints, is therefore defined as follows.

Definition 8 (Transition relation). Let $H_{k}=h_{1}, \ldots, h_{j}, H_{r}=h_{j+1}, \ldots, h_{n}$ and $C=d_{1}, \ldots, d_{m}$ denote conjunctions of $C H R$ constraints, let $G$ and $B$ denote conjunctions of built-in constraints, let $\sigma$ be a match substitution for the heads of the rule $R$ and let $\theta$ be a c.a.s. for built-ins in the guard. Then, $\rightarrow$ is:

\begin{tabular}{|c|}
\hline Solve \\
\hline $\begin{array}{l}\boldsymbol{I F} S=b \cup S^{\prime}, \text { where } b \text { is a built-in constraint such that } C T \models \exists \theta: b \theta \\
\boldsymbol{T H E N}\langle S, T\rangle_{\nu} \stackrel{C T \theta}{\longrightarrow}\left\langle S^{\prime} \theta, T\right\rangle_{\nu}\end{array}$ \\
\hline Simplify \\
\hline $\begin{array}{c}\text { IF }\left(R_{s} @ H_{k} \backslash H_{r} \Leftrightarrow G \mid B, C\right) \text { is a fresh variant of a rule in } P \\
\text { and } S=H_{k}^{\prime} \cup H_{r}^{\prime} \cup S^{\prime}, \text { with } \\
H_{k}^{\prime}=\left\{c_{1} \sharp i_{1}, \ldots, c_{j} \sharp i_{j}\right\} \text { and } H_{r}^{\prime}=\left\{c_{j+1} \sharp i_{j+1}, \ldots, c_{n} \sharp i_{n}\right\} \\
\text { such that } C T \models \exists \sigma \theta:\left(c_{1}=h_{1} \sigma\right) \wedge \cdots \wedge\left(c_{n}=h_{n} \sigma\right) \wedge G \sigma \theta \\
\boldsymbol{T H E N}\langle S, T\rangle_{\nu} \stackrel{R_{s} \sigma \theta}{\longrightarrow}\left\langle S^{\prime \prime}, T^{\prime \prime}\right\rangle_{\nu+m} \\
\text { where } S^{\prime \prime}=\left(H_{k}^{\prime} \cup S^{\prime} \cup B \cup\left\{d_{1} \sharp \nu, \ldots, d_{m} \sharp(\nu+m-1)\right\}\right) \sigma \theta \text { and } \\
\text { where } T^{\prime \prime}=\left(\left(T \backslash T_{\left(H_{r}^{\prime}, T\right)}^{E}\right) \cup T_{\left(\left\{d_{1} \sharp \nu, \ldots, d_{m} \sharp(\nu+m-1)\right\}, H_{k}^{\prime} \cup S^{\prime}\right)}^{A}\right)\end{array}$ \\
\hline Propagate \\
\hline $\begin{array}{l}\boldsymbol{I F}\left(R_{p} @ H_{k} \Rightarrow G \mid B, C\right) \text { is a fresh variant of a rule in } P \\
\quad \text { and } S=H_{k}^{\prime} \cup S^{\prime}, \text { with } H_{k}^{\prime}=\left\{c_{1} \sharp i_{1}, \ldots, c_{j} \sharp i_{j}\right\} \\
\quad \text { and } T=\left\{\left(R_{p}, i_{1}, \ldots, i_{j}\right)\right\} \cup T^{\prime} \\
\quad \text { such that } C T \models \exists \sigma \theta:\left(c_{1}=h_{1} \sigma\right) \wedge \cdots \wedge\left(c_{j}=h_{j} \sigma\right) \wedge G \sigma \theta \\
\text { THEN }\langle S, T\rangle_{\nu} \stackrel{R_{p} \sigma \theta}{\longrightarrow}\left\langle S^{\prime \prime}, T^{\prime \prime}\right\rangle_{\nu+m} \\
\text { where } S^{\prime \prime}=\left(S \cup B \cup\left\{d_{1} \sharp \nu, \ldots, d_{m} \sharp(\nu+m-1)\right\}\right) \sigma \theta \text { and } \\
\text { where } T^{\prime \prime}=\left(T^{\prime} \cup T_{\left(\left\{d_{1} \sharp \nu, \ldots, d_{m} \sharp(\nu+m-1)\right\}, S\right)}^{A}\right)\end{array}$ \\
\hline
\end{tabular}

Note that the label represented by $\nu$ is an integer assigned to the first constraint in $C$ that is added to the constraint store. Then, $\nu+1$ is assigned to the second added constraint and so on.

The definition for the transition relation states that for a rule to be applicable there must exist matching CHR constraints in the constraint store for which the guard can be satisfied. 
Example 2 (Executing Primes). We revisit Primes from Example 1 and execute it with a query $\left\langle\{\text { primes }(7) \sharp 1\},\left\{\left(R_{2}, 1\right)\right\}\right\rangle_{2}$. We get as a possible computation:

$$
\begin{aligned}
& I_{0}=\quad\left\langle\{\operatorname{primes}(7) \sharp 1\},\left\{\left(R_{2}, 1\right)\right\}\right\rangle_{2} \underset{R_{2}}{\stackrel{R_{2}}{\longrightarrow}} \\
& I_{1}=\quad\left\langle\{\text { primes }(7) \sharp 1, \text { primes }(6) \sharp 2\},\left\{\left(R_{2}, 2\right)\right\}\right\rangle_{3} \stackrel{R_{2}}{\longrightarrow} \\
& I_{2}=\quad\left\langle\{\text { primes }(7) \sharp 1, \text { primes }(6) \sharp 2, \text { primes }(5) \sharp 3\},\left\{\left(R_{2}, 3\right)\right\}\right\rangle_{4} \stackrel{R_{2}}{\longrightarrow} \\
& I_{3}=\quad\left\langle\{\operatorname{primes}(7) \sharp 1, \operatorname{primes}(6) \sharp 2, \operatorname{primes}(5) \sharp 3, \text { primes }(4) \sharp 4\},\left\{\left(R_{2}, 4\right)\right\}\right\rangle_{5} \stackrel{R_{2}}{\longrightarrow} \\
& I_{4}=\left\langle\{\operatorname{primes}(7) \sharp 1, \operatorname{primes}(6) \sharp 2, \operatorname{primes}(5) \sharp 3, \operatorname{primes}(4) \sharp 4, \operatorname{primes}(3) \sharp 5\},\left\{\left(R_{2}, 5\right)\right\}\right\rangle_{6} \stackrel{R_{1}}{\longrightarrow} \\
& I_{5}=\quad\left\langle\{\operatorname{primes}(7) \sharp 1, \operatorname{primes}(5) \sharp 3, \text { primes }(4) \sharp 4, \text { primes }(3) \sharp 5\},\left\{\left(R_{2}, 5\right)\right\}\right\rangle_{6} \stackrel{R_{2}}{\longrightarrow} \\
& I_{6}=\quad\langle\{\operatorname{primes}(7) \sharp 1, \operatorname{primes}(5) \sharp 3, \operatorname{primes}(4) \sharp 4, \operatorname{primes}(3) \sharp 5, \operatorname{primes}(2) \sharp 6\}, \emptyset\rangle_{7} \stackrel{R_{1}}{\longrightarrow} \\
& I_{7}=\quad\langle\{\text { primes }(7) \sharp 1, \text { primes }(5) \sharp 3, \text { primes }(3) \sharp 5, \operatorname{primes}(2) \sharp 6\}, \emptyset\rangle_{7}
\end{aligned}
$$

Note that we have omitted discussion of the solve transition. We assume in this example that built-ins are solved immediately.

\subsection{Solving built-in constraints}

Solve transitions in computations introduce bindings. These can be delayed, however. Whether bindings caused by such built-ins are available at the time CHR constraints cause rules to fire, is therefore uncertain. In some cases however, delaying a built-in causes a CHR constraint to be delayed as well. The next example revisits a rule of Primes in Example 1 in which this is the case.

Example 3. generate@ $\operatorname{primes}(N) \Rightarrow N>2 \mid N p$ is $N-1$, primes $(N p)$.

When adding the built-in $N p$ is $N-1$, it can be noted, given the guard $N>2$, that the added CHR constraint primes $(N p)$ can only fire the rule again when the built-in has been solved. That is, the constraints matching with $\operatorname{primes}(N)$ in the head of the rule have to be ground. Therefore, in this case, we can use the built-in to infer interargument relations.

By analyzing which constraints can fire rules, we know to what extent argument positions will be instantiated. By considering therefore a call set and a rigid interpretation in this call set, we can use built-ins, as we only measure argument positions which will be instantiated enough. As such, only interargument relations from added built-ins of which we know these must introduce bindings, are taken into account. When measuring sizes of added built-ins, we assign the level value 0 . After all, we assume them to terminate on their own.

\subsection{CHR computations}

In CHR, a transition is often called a computation step and a sequence of computation steps is called a computation. For a query $I$ and a program $P$, there are usually several different possible computations.

Termination of CHR programs executed under a theoretical semantics corresponds to the notion of universal termination, where we require finiteness of all computations originating from a query.

Definition 9 (Termination of a CHR program). We say that a CHR program $P$ terminates for a query I iff all computation of $P$ for $I$ are finite. 
Without loss of generality, we regard computations in CHR as a subsequence of simplification steps, interleaved with sequences of zero or more propagation steps. Computations are therefore of the following form,

$$
s_{(1,1)} \stackrel{R_{p_{(1,1)}}}{\longrightarrow} s_{(1,2)} \stackrel{R_{p_{(1,2)}}}{\longrightarrow} \ldots \stackrel{R_{s_{1}}}{\longrightarrow} s_{(2,1)} \stackrel{R_{p_{(2,1)}}}{\longrightarrow} s_{(2,2)} \stackrel{R_{p_{(2,2)}}}{\longrightarrow} \ldots \stackrel{R_{s_{2}}}{\longrightarrow} \ldots
$$

where $R_{p_{(i, j)}}$ represents the application of a propagation rule and $R_{s_{i}}$ that of a simplification rule. Note that a propagation sequence may be infinitely long.

\subsection{The propagation store}

Propagation in CHR computations serves as a way to complete state information. When the state after simplification is propagated upon, we reach some more completed state on which we can further simplify.

W.r.t. propagation we distinguish two different kinds of CHR states. A fully propagated state is a state with an empty token store. Therefore, no propagation can take place on a fully propagated state. A partially propagated state is a state which does contain tokens. We define the action of full propagation and partial propagation, where we refer to a sequence of propagation steps, originating from a CHR state and ending in a fully or partially propagated state, respectively.

When fully propagating on a state, independent of the order in which propagation rules are applied, we end up in an equivalent state. Such equivalent states contain the same constraints. However, they may be labeled differently as propagation rules are applied in different orders.

Given a state, we refer to the propagation store as the multiset of all the constraints that are added when fully propagating on the given state. Obviously, the constraints added by partially propagating on a state correspond to a subset of the propagation store as applying a propagation rule now corresponds to the introduction of constraints from the propagation store to the constraint store. Up to identification, their union therefore remains constant under propagation.

Example 4 (Executing Primes). We revisit the computation of Primes from Example 2 and represent for it the propagation store:

$$
\begin{aligned}
& \left\langle\{\text { primes }(7) \sharp 1\},\{\operatorname{primes}(6), \operatorname{primes}(\mathbf{5}), \operatorname{primes}(4), \operatorname{primes}(3), \operatorname{primes}(2)\},\left\{\left(R_{2}, 1\right)\right\}\right\rangle_{2} \\
& \left\langle\{\text { primes }(7) \sharp 1, \text { primes }(6) \sharp 2\},\{\operatorname{primes}(5), \operatorname{primes}(\mathbf{4}), \operatorname{primes}(\mathbf{3}), \operatorname{primes}(\mathbf{2})\},\left\{\left(R_{2}, 2\right)\right\}\right\rangle_{3} \\
& \left\langle\{\text { primes }(7) \sharp 1, \text { primes }(6) \sharp 2, \text { primes }(5) \sharp 3\},\{\operatorname{primes}(\mathbf{4}), \operatorname{primes}(\mathbf{3}), \operatorname{primes}(\mathbf{2})\},\left\{\left(R_{2}, 3\right)\right\}\right\rangle_{4} \\
& \left\langle\{\text { primes }(7) \sharp 1, \text { primes }(6) \sharp 2, \text { primes }(5) \sharp 3, \text { primes }(4) \sharp 4\},\{\operatorname{primes}(\mathbf{3}) \text {, primes }(2)\},\left\{\left(R_{2}, 4\right)\right\}\right\rangle_{5} \\
& \begin{array}{r}
\left\langle\{\text { primes }(7) \sharp 1, \text { primes }(6) \sharp 2, \text { primes }(5) \sharp 3, \text { primes }(4) \sharp 4, \text { primes }(3) \sharp 5\},\{\text { primes }(2)\},\left\{\left(R_{2}, 5\right)\right\}\right\rangle_{6} \\
\left\langle\{\text { primes }(7) \sharp 1, \text { primes }(5) \sharp 3, \text { primes }(4) \sharp 4, \text { primes }(3) \sharp 5\},\{\text { primes }(2)\},\left\{\left(R_{2}, 5\right)\right\}\right\rangle_{6}
\end{array} \\
& \langle\{\text { primes }(7) \sharp 1, \text { primes }(5) \sharp 3 \text {, primes }(4) \sharp 4 \text {, primes }(3) \sharp 5 \text {, primes }(2) \sharp 6\},\{\},\{\}\rangle_{7} \\
& \langle\{\text { primes }(7) \sharp 1, \text { primes }(5) \sharp 3, \text { primes }(3) \sharp 5, \text { primes }(2) \sharp 6\},\{\},\{\}\rangle_{7}
\end{aligned}
$$

Note that we have left out state names as well as transitions due to space restrictions. The propagation store together with the constraint store remains constant when propagation occurs. When simplifying, their combined size decreases. It turns out that this is typical for terminating programs.

A problem regarding the propagation store is that it can be infinitely large for some states in computations of a CHR program. To prevent this, we formulate conditions on propagation rules that guarantee that no sequence of propagation rules in computations of a CHR program can be infinitely long. Since such a condition is related to termination, we discuss it in the next section. 


\section{Termination of general CHR programs}

Due to CHR's multi-headed rules and multiset semantics, it is often difficult to predict the behavior of CHR programs. Especially the concept of propagation, complicates reasoning about program properties, since it requires a fire-once policy on propagation rules to prevent trivial non-termination. New concepts are therefore required to handle propagation, which cannot be directly adapted from existing notions in LP an TRS termination analysis.

Example 5. Consider the following propositional CHR program,

$$
R_{1} @ a, a \Rightarrow b . \quad R_{2} @ b, b, b \Leftrightarrow a .
$$

For a query with two constraints $a$, the program terminates as $R_{1}$ can only be applied in two different ways, as such only adding 2 constraints $b$. However, when querying with three $a$ 's, the propagation rule can be applied in 6 different ways. As a consequence, the second rule becomes applicable twice, as such causing new tokens to be introduced. It is therefore a non-terminating program.

Currently, two approaches exist, one for CHR without propagation and another for CHR with propagation. The first one, essentially adapts existing conditions in LP and TRS. It measures the size of the constraint store and guarantees decreases between consecutive CHR states. The second approach formulates conditions on adding constraints to the constraint store. In order to be able to handle propagation, it is strictly less powerful on CHR programs without propagation.

Although the approaches can trivially be combined into a global approach (by using one condition for simplification only programs, the other for programs with propagation), it would be satisfactory to formulate general conditions. In addition, this turns out to gain a new class of programs we can prove terminating.

Example 6 (Problem class). The program is a constructed example, representing a class of CHR programs which cannot be proved terminating using existing approaches. It contains both a propagation rule and a simplification rule and terminates for all finite queries.

$$
R_{1} @ a(s(N)), a(N), a(N) \Leftrightarrow a(s(N)), a(N) . \quad R_{2} @ a(s(s(N))) \Rightarrow a(N) .
$$

As the program contains a propagation rule, only the condition for CHR with propagation can be used to prove termination. However, as the condition for CHR with propagation requires that $|a(s(s(N)))|>|a(N)|$ for the propagation rule, a decrease in the number of maximally ranked constraints in the simplification rule can never be shown.

In this section, we present a general approach, capable of proving termination of an entirely new class of CHR programs of the kind as presented in the example above. Our conditions are strictly more powerful than those formulated in the existing approaches. We resolve therefore the drawbacks of existing approaches. 


\subsection{Preliminaries}

First, we define some preliminary notions w.r.t. interpretations for constraints in CHR programs. These notions are adapted from the LP context [4-6]. Then, we introduce the existing approaches $[11,16]$.

Term $_{P}$ and $\operatorname{Con}_{P}$ denote the sets of respectively all terms and all constraints that can be constructed from the alphabet underlying $P$. As in LP, we wish to describe the constraints that participate in computations of a CHR program $P$, given the constraints $S$ that are part of the query, by its call set. As such, we can establish interpretations which result in better approximations of the behavior of a CHR program.

Definition 10 (Call set). Let $S \subseteq \operatorname{Con}_{P}$. Then by $\operatorname{Call}(P, S)$, we denote the subset of $C_{P o n}$, such that $C \in C$ all $(P, S)$ whenever $C$ is a constraint used to apply a rule in some computation of $P$ for $I$, where $I \subseteq S$.

To measure constraints, we use norms and level mappings. In general this is referred to as interpretations for CHR constraints. The sizes of consecutive computation states are compared using the level values (or ranks) of the constraints in the state. We recall their definition here in the context of CHR.

Definition 11 (Norm, level mapping). Let $P$ be a CHR program. Then, a norm is a function $\|\|:$. Term $_{P} \rightarrow \mathbb{N}$ and a level mapping $||:$. Con $_{P} \rightarrow \mathbb{N}$.

Several examples of norms and level mappings can be found in literature on LP termination analysis [5]. Two well-known norms are list-length and termsize. The most common kind of level mapping is the linear level mapping, where a constraint is mapped to a positive linear combination of the positive integer norms of its terms. We also require norms and level mappings to be rigid w.r.t. the constraints represented by the call set. That is, all constraints in the call set must have unique interpretations, which cannot alter under substitution.

Termination of CHR programs without propagation. In [11], a condition for CHR programs without propagation is discussed. In such a setting, decreases are shown between consecutive CHR states. These are compared by using a multiset order [7] on the constraint store. If such a decrease is shown for every application of a rule, the program must terminate.

Definition 12 (Multiset order). Let $r$ represent the level value of some atom and let $n_{r}^{s}$ represent the number of atoms of level $r$ in a multiset $s$. Then a multiset order is an induced order, given a level mapping |.| for its atoms. A multiset $s$ is considered larger than a multiset $t$, denoted $s \succ_{m} t$, if some atom of level value $r$ exists, such that $n_{r}^{s}>n_{r}^{t}$ and such that $\forall q>r: n_{q}^{s}=n_{q}^{t}$.

The next example demonstrates how to prove termination of a CHR program with a multiset order on the constraint store.

Example 7. The program is terminating for all ground queries.

$$
R_{1} @ a(s(N)), a(N), a(N) \Leftrightarrow a(s(N)), a(N) . \quad R_{2} @ a(s(N)) \Leftrightarrow a(N) .
$$

Termination is shown using a level mapping $|a(N)|=\|N\|$ and multiset order. 
Termination of CHR programs with propagation. The ranking condition (RC) for CHR with propagation proves termination in an entirely different way [16]. It guarantees that only a finite number of constraints can be added to the constraint store. Because simplification removes constraints and propagation respects the fire-once policy, this implies termination.

The RC compares sizes of individual constraints, rather than multisets of constraints. It requires that propagation rules can only add constraints ranked strictly lower than any of the head constraints that gave cause to it. For simplification rules the number of constraints removed of maximal rank, has to be strictly greater than those added of maximal rank.

Example 8. The following example demonstrates the use of the $\mathrm{RC}$ for $\mathrm{CHR}$ with propagation.

$$
R_{1} @ a(s(s(N))) \Rightarrow a(s(N)), a(N) . \quad R_{2} @ a(s(N)) \Leftrightarrow a(N) .
$$

Termination is shown for ground queries, using a level mapping from constraints $|a(N)|=\|N\|$ to the set of natural numbers. As such, a decrease in maximally ranked constraint exists in the second rule, while the first rule only adds constraints which are ranked strictly lower than those which fired the rule.

Notice that the condition on simplification is a strengthened case of multiset

order. Therefore, in the case of a program with only simplification rules, the condition for CHR without propagation covers more programs.

Example 9. We revisit Example 7.

$$
R_{1} @ a(s(N)), a(N), a(N) \Leftrightarrow a(s(N)) . \quad R_{2} @ a(s(N)) \Leftrightarrow a(N) .
$$

Termination cannot be shown as the second rule requires that $|a(s(N))|>$ $|a(N)|$. As such, no decrease in maximally ranked constraint can be shown for the first rule. The reason is that in the case of a propagation rule, e.g. $R_{2} @ a(s(N)) \Rightarrow a(N)$, we obtain a non-terminating program.

\subsection{Termination of general CHR programs}

As mentioned earlier, we can regard a computation in CHR as a subsequence of simplification steps, interleaved with sequences of propagation steps. A CHR program can only be guaranteed to terminate if these sequences of propagation steps cannot be infinitely long. The RC on propagation rules guarantees this.

Definition 13 (RC on propagation rules). Let $R_{p} @ h_{1}, \ldots, h_{n} \Rightarrow G$ $b_{1}, \ldots, b_{m}$ be a propagation rule in a CHR program $P, I$ a query and $\sigma$ a match substitution for the heads of $R_{p}$ such that $C T \models \exists \theta: G \sigma \theta$ holds. Then, the $R C$ on propagation rules is satisfied w.r.t. a rigid level mapping $|$.$| for \operatorname{Call}(P, I)$ iff $\forall h_{i}, b_{j}:\left|h_{i} \sigma \theta\right|>\left|b_{j} \sigma \theta\right|$. 
At first sight, the condition on propagation seems unnecessarily strict. However, if we would not require that all body constraints are ranked strictly lower than any of the head constraints, it is possible that added constraints replace those which gave cause to them, enabling the rule to be fired at the same level of interpretation. We illustrate the RC by proving termination of the following example program without simplification rules.

Example 10 (Fibonacci). The program calculates Fibonacci numbers. The first rules resolve base cases, while the third rule adds Fibonacci constraints.

$$
\begin{aligned}
& R_{1} @ f i b(N, M) \Rightarrow N=0 \mid M=0 . \\
& R_{2} @ f i b(N, M) \Rightarrow N=s(0) \mid M=1 . \\
& R_{3} @ f i b\left(s(s(N)), M_{1}\right), f i b\left(s(N), M_{2}\right) \Rightarrow f i b(N, M), M_{1} \text { is } M_{2}+M .
\end{aligned}
$$

As we will query the program with constraints of the type $f i b(n, m)$ with $n$ ground and $m$ a variable, we measure these constraints by the term-size of their first argument. Therefore, $|f i b(N, M)|=\|N\|_{t s}$ is rigid w.r.t. the call set. The first rules trivially satisfy the $\mathrm{RC}$ as no CHR constraints are added. As for the third rule the first argument decreases in term-size, we prove termination.

The next proposition formulates that when the $\mathrm{RC}$ is satisfied for all propagation rules in a CHR program, there cannot exist infinite sequences of propagation steps in computations for the program.

Proposition 1. If a CHR program $P$ with a query I satisfies the $R C$ on propagation rules w.r.t. a rigid level mapping |.|, then there cannot exist infinite propagation in a computation of $P$, if the constraint store $S$ is finite.

Proof. We rank tokens according to the corresponding CHR constraint of smallest rank. Therefore, no token added by propagation can be of equal size or greater than the token removed. This implies that the multiset size of the token store decreases after every application of a propagation rule. No infinite sequences of propagation steps can therefore exist.

When a CHR program satisfies the $\mathrm{RC}$ on propagation rules, then in any CHR state in a computation of the program, the propagation store is finite.

Corrolary 1 If a CHR program $P$ with a query I satisfies the $R C$ on propagation rules w.r.t. a level mapping |.|, then for any CHR state in a computation of $P$ for $I$, the propagation store $V$ is finite if the constraint store $S$ is finite.

We now come to the heart of our approach. As we could already observe in Example 4, when propagating, the combined size of constraint and propagation store $|\operatorname{chr}(S) \uplus V|$ remains equal while the size of the token store $|T|$ decreases. We define therefore the following lexicographical interpretation for CHR states.

Definition 14 (CHR state interpretation). The CHR state interpretation, is a lexicographical order of the following form: 


$$
\left|\langle S, T\rangle_{\nu}\right|=\langle|\operatorname{chr}(S) \uplus V|,|T|\rangle
$$

Here, $\uplus$ denotes multiset union, $\operatorname{chr}(S)=\{\operatorname{chr}(C) \mid C \in S\}$ the unlabeled variant of the constraint store, $V$ the propagation store and $T$ the token store. $\operatorname{chr}(S)$ and $V$ are multisets of constraints. The tokens are measured by the smallest level value of a CHR constraint represented in the token: $\left|\left(R_{i}, i d_{1}, \ldots, i d_{n}\right)\right|=$ $\min \left\{\left|\operatorname{chr}\left(c_{1} \sharp i d_{1}\right)\right|, \ldots,\left|\operatorname{chr}\left(c_{n} \sharp i d_{n}\right)\right|\right\}$.

We formulate a theorem stating that termination of a CHR program is guaranteed, when decreases are found in the CHR state interpretation, as defined above, for every application of a rule in a CHR program.

Theorem 1. Let $P$ be a CHR program and I a query. Furthermore, let |.| be a rigid level mapping for the constraints in $\operatorname{Call}(P, I)$. Then, a CHR program $P$ terminates for $I$ if for all applications of rules in $P$, using only constraints in $\operatorname{Call}(P, I)$, the lexicographical ordering on CHR states decreases.

Proof. The ordering we use is well-founded. Therefore, computations can only be finitely long. By definition, this implies termination of $P$.

Note that whenever the $\mathrm{RC}$ on propagation rules is satisfied, we guarantee that there is a decrease in the CHR state interpretation for every application of a propagation rule. To observe decreases when simplifying on states, we must guarantee decreases in $|\operatorname{chr}(S) \uplus V|$ as the token store may increase in size. Such decreases can be observed in Example 4 as well.

To formulate a condition on simplification rules, which guarantees decreases in $|\operatorname{ch} r(S) \uplus V|$, we first guarantee that a multiset decrease exists in the constraint store $\operatorname{chr}(S)$. Such a decrease can be shown using the condition of Frühwirth [11].

Definition 15 (RC on simplification rules). Let $R_{s} @ h_{(s, 1)}, \ldots, h_{\left(s, j_{s}\right)} \backslash$ $h_{\left(s, j_{s}+1\right)}, \ldots, h_{\left(s, n_{s}\right)} \Leftrightarrow G_{s} \mid b_{(s, 1)}, \ldots, b_{\left(s, m_{s}\right)}$ be a simplification rule in a CHR program $P$. Let $\sigma$ be a match substitution for the head constraints such that $C T \models \exists \theta: G_{s} \sigma \theta$ holds and let |.| be a rigid level mapping w.r.t. a CHR program $P$ and a query $I$, such that the added and removed constraints in $R_{s}$ have ranks $r_{1}>r_{2}>\ldots>r_{k}$ and such that $n_{i}^{a}$ and $n_{i}^{r}$ represent respectively the number of constraints of rank $r_{i}$ added and removed by $R_{s}$. Then, $R_{s}$ satisfies the $R C$ on simplification rules w.r.t. |.| iff $\exists r_{j}: n_{j}^{r}>n_{j}^{a}$ and $\forall r_{i}>r_{j}: n_{j}^{r}=n_{j}^{a}$.

We already illustrated the RC in Example 7 on a program without propagation. However, when propagation is present, simplification rules cannot be considered separately. After all, by propagating on the added constraints of the simplification rule, the decrease caused in $\operatorname{chr}(S)$ can still be undone. The constraints that can be added by propagation are represented in $V$. As a consequence, we have to refine our condition on simplification rules to guarantee that none of the constraints added to $V$ can undo the decrease caused in $\operatorname{chr}(S)$. It is however impractical to analyze this multiset of added constraints.

Therefore, we observe the following. By definition, the tokens that enter the token store correspond to added constraints in the simplification rule. These 
tokens can result in the addition of new constraints and thus new tokens. This process dies out when the RC on propagation rules is satisfied.

The application of a propagation rule is in one-to-one correspondence with a token. Given all tokens in the token store, we can apply their corresponding propagation rules simultaneously. The resulting multiset of added constraints from applying these rules simultaneously is referred to as the layer 1 constraints and is denoted by the multiset $L_{1}$. By adding these constraints, new tokens are added, resulting as such in layer two constraints $L_{2}$. For a finite propagation store, there are only a finite number of such layers: $V=L_{1} \uplus L_{2} \uplus \cdots \uplus L_{v}$.

Given the RC on propagation rules, we know that if constraints in $L_{1}$ cannot undo the multiset decrease in the constraint store that none of the subsequent layers $L_{i}$ can undo it either. This is because, if the added constraints in the first layer are already smaller than the relevant ranks in the simplification rule, than the added constraints in next layers need to be even smaller. Thus, they can definitely not influence the relevant ranks of the simplification rule. It is therefore sufficient to only consider $L_{1}$ constraints.

Definition 16 (Refined RC on simplification rules). Consider a simplification rule $R_{s} @ h_{(s, 1)}, \ldots, h_{\left(s, j_{s}\right)} \backslash h_{\left(s, j_{s}+1\right)}, \ldots, h_{\left(s, n_{s}\right)} \Leftrightarrow G_{s} \mid b_{(s, 1)}, \ldots, b_{\left(s, m_{s}\right)}$ in a CHR program $P$ that satisfies the $R C$ on simplification rules for I w.r.t. a rigid level mapping |.|. Therefore, $\exists r_{j}: n_{j}^{r}>n_{j}^{a}$ and $\forall r_{i}>r_{j}: n_{j}^{r}=n_{j}^{a}$. Then, the refined $R C$ on simplification rules is satisfied for $R_{s}$ iff for all heads $h_{\left(p, i_{p}\right)}$ in propagation rules in $P$ for which $C T \models \exists \mu \sigma^{\prime} \theta^{\prime}:\left(G_{s} \sigma \theta \wedge\left(b_{\left(s, i_{s}\right)} \sigma \theta=\right.\right.$ $\left.\left.h_{\left(p, i_{p}\right)} \mu\right) \wedge G_{p} \mu \sigma^{\prime} \theta^{\prime}\right)$ holds: $r_{j}>\left|b_{(p, k)} \mu \sigma^{\prime} \theta^{\prime}\right|$ for all $b_{(p, k)}$. Here, $b_{(p, k)}$ is an added constraint in the propagation rule and $\mu$ a substitution for matching $b_{\left(s, i_{s}\right)} \sigma \theta$ with $h_{\left(p, i_{p}\right)}$. The substitutions $\sigma \theta$ come from matching and answer substitutions in the simplification rule and $\sigma^{\prime}$ is a match substitution for the heads of the propagation rule and $\theta^{\prime}$ a c.a.s. for satisfaction of the guard $G_{p}$ of the propagation rule.

We illustrate the refined $\mathrm{RC}$ by proving termination of the problem class from Example 6.

Example 11 (Problem class). The program terminates for ground queries.

$$
R_{1} @ a(s(N)), a(N), a(N) \Leftrightarrow a(s(N)) . \quad R_{2} @ a(s(s(M))) \Rightarrow a(M) .
$$

By the $\mathrm{RC}$ on propagation rules, we require that $|a(s(s(M)))|>|a(M)|$. This is satisfied if we measure constraints by a term-size norm: $|a(N)|=\|N\|$. By the refined $\mathrm{RC}$ on simplification rules, we have that the constraints of rank $|a(N)|$ are decreased in number. By propagation on the added constraints this number cannot be increased again: $|a(M)|<|a(N)|$ when $a(s(N))=a(s(s(M)))$.

Proposition 2. If a CHR program $P$ satisfies the refined $R C$ for simplification rules w.r.t. a rigid level mapping $|$.$| for C a l l(P, I)$, then there exists no infinite subsequence of simplification rules in a computation for $P$ with $I$.

Proof. By the RC for simplification we know that $\exists r_{j}: n_{j}^{r}>n_{j}^{a}$ and $\forall r_{i}>$ $r_{j}: n_{j}^{r}=n_{j}^{a}$. There is therefore a decrease in the size of the constraint store. 
By considering the added constraints by propagating on the obtained state, we guarantee that the added constraints to the propagation store by simplification cannot undo this decrease. As such, there can only exist a finite subsequence of simplification steps in computations for $P$ with $I$.

By Proposition 1 and 2, we guarantee decreases for every application of a rule in a $\mathrm{CHR}$ program if the $\mathrm{RC}$ on propagation rules and the refined $\mathrm{RC}$ on simplification rules are satisfied. Therefore, the CHR program must terminate.

Theorem 2. A CHR program $P$ terminates for a query $I$ if it satisfies the $R C$ for propagation and the refined $R C$ for simplification w.r.t. a rigid level-mapping |.| for $\operatorname{Call}(P, I)$.

Proof. By Proposition 1 and 2, a decrease in state size can be detected for each rule application. By Theorem 1, $P$ must terminate for $I$.

\section{Discussion}

We evaluate our method first by comparing it to existing approaches. As stated earlier the condition on CHR without propagation requires a multiset decrease. In that setting, our method is as strong for the considered class of CHR programs.

Proposition 3. The RC for CHR programs without propagation [11] is a special case of the RC for general CHR programs.

W.r.t. the approach taken for CHR with propagation, our approach is more general as well. The condition formulated on propagation rules is identical to ours. However, our condition on simplification rules is more general. Our method also allows lower ranked constraints to be evaluated.

Proposition 4. The RC for CHR programs with propagation [16] is a special case of the RC for general CHR programs.

Therefore, our approach is able to prove strictly more programs terminating. w.r.t. both approaches. Consider the next example program.

Example 12. $\quad R_{1} @ \operatorname{list}([D \mid L]), \operatorname{list}(L) \Leftrightarrow \operatorname{list}([D \mid L])$.

$$
\begin{aligned}
& R_{2} @ \operatorname{list}\left(\left[A, B, C \mid L_{1}\right]\right), \operatorname{list}\left(\left[A, B, C \mid L_{2}\right]\right) \Rightarrow \\
& \quad \text { length }\left(L_{1}, S_{1}\right), \text { length }\left(L_{2}, S_{2}\right), S_{1}=S_{2} \mid \operatorname{list}\left(L_{1}\right), \operatorname{list}\left(L_{2}\right) .
\end{aligned}
$$

Termination cannot be proved when using the existing approaches. Our approach can prove termination for queries with nil-terminated lists. As such the constraints in the call set can be measured by a rigid level mapping, list-length. In case of the propagation rule, the condition states that: $\forall h_{i}, b_{j}:\left|h_{i} \sigma \theta\right|>\left|b_{j} \sigma \theta\right|$.

$$
\begin{array}{ll}
\left|\operatorname{list}\left(\left[A, B, C \mid L_{1}\right]\right)\right|>\left|\operatorname{list}\left(L_{1}\right)\right| & \left|\operatorname{list}\left(\left[A, B, C \mid L_{2}\right]\right)\right|>\left|\operatorname{list}\left(L_{1}\right)\right| \\
\left|\operatorname{list}\left(\left[A, B, C \mid L_{1}\right]\right)\right|>\left|\operatorname{list}\left(L_{2}\right)\right| & \left|\operatorname{list}\left(\left[A, B, C \mid L_{2}\right]\right)\right|>\left|\operatorname{list}\left(L_{2}\right)\right|
\end{array}
$$


For list-length these conditions are satisfied.

For the first rule, the number of constraints of rank $|\operatorname{list}([D \mid L])|$ remains the same and the number of constraints of rank $|\operatorname{list}(L)|$ is decreased in number. Now, we verify that none of the constraints added by propagating on the added constraint list $([D \mid L])$ can undo this decrease. That is,

$$
|\operatorname{list}(L)|>\left|\operatorname{list}\left(L_{1}\right)\right| \text { and }|\operatorname{list}(L)|>\left|\operatorname{list}\left(L_{2}\right)\right|
$$

This is a consequence of matching the body of the simplification rule with the heads of the propagation rule: $[D \mid L]=\left[A, B, C \mid L_{1}\right]$ and $[D \mid L]=\left[A, B, C \mid L_{2}\right]$. Therefore, the number of constraints of maximally rank $|\operatorname{list}([D \mid L])|$ remains the same, the number of constraint of rank $|\operatorname{list}(L)|$ decreases. Both RCs are therefore satisfied w.r.t. list-length. The program therefore terminates for all queries of constraints with nil-terminated lists.

Our approach is currently unable to handle the following kind of programs:

Example 13. The program terminates for ground queries.

$$
R_{1} @ a(s(N)), a(N), a(N) \Leftrightarrow a(s(N)) . \quad R_{2} @ a(s(N)) \Rightarrow a(N) .
$$

Note that the program terminates because the simplification rule removes two constraints of rank $r_{j}$, while the propagation rule only adds one such constraint.

The refined $\mathrm{RC}$ on simplification rules cannot be satisfied for this program. The constraints of rank $|a(N)|$ are decreased in number in the first rule while the constraints added by propagating on the added constraints of the simplification rule are of the same rank. That is, $r_{j}=\left|b_{(p, k)} \mu \sigma^{\prime} \theta^{\prime}\right|$.

For single-headed propagation rules, we can however refine our condition by considering that no recombinations are required to fire these rule. Because of this direct correspondence, we do not have to assume that multiple instances of some constraint can be added. Therefore, for every added CHR constraint in rule 1, we add only one constraint by the propagation rule. The decrease caused in the constraint store is therefore not undone by propagation. In such cases, we can allow that $r_{j}=\left|b_{(p, k)} \mu \sigma^{\prime} \theta^{\prime}\right|$, given that the combined effect of multiple single-headed propagation rules does not undo the decrease.

We will study this refinement in future work.

\section{Conclusion}

In this paper, we presented a new approach to termination analysis of CHR programs. Our approach proves termination by formulating conditions on the size of a CHR state. To measure states, we introduced a new interpretation, based on the constraint store, the token store and the propagation store. On the basis of these we compose a lexicographical interpretation and formulate conditions guaranteeing decreases between all consecutive CHR states. We showed that our approach generalizes the existing approaches and that it is able to prove termination of an entirely new class of CHR programs. In future work, we will refine the approach for single-headed propagation rules. We will develop an efficient termination tool to support thorough experimentation. 


\section{References}

1. S. Abdennadher. Operational semantics and confluence of constraint propagation rules. In $C P 97$, pages 252-266. Springer-Verlag, 1997.

2. S. Abdennadher, T. Frühwirth, and H. Meuss. On confluence of constraint handling rules. In CP96. Springer-Verlag, 1996.

3. S. Abdennadher and M. Marte. University course timetabling using constraint handling rules. Applied Artificial Intelligence, 14(4):311-325, 2000.

4. M. Bezem. Characterizing termination of logic programs with level mappings. In NACLP, pages 69-80, 1989.

5. D. De Schreye and S. Decorte. Termination of logic programs: the never-ending story. Journal of Logic Programming, 19-20:199-260, 1994.

6. N. Dershowitz. Termination of rewriting. Journal of Symbolic Computation, 3(12):69-116, 1987.

7. N. Dershowitz and Z. Manna. Proving termination with multiset orderings. Commun. ACM, 22(8):465-476, 1979.

8. G.J. Duck, P.J. Stuckey, M.J. García de la Banda, and C. Holzbaur. The refined operational semantics of constraint handling rules. In ICLP04, pages 90-104, 2004.

9. G.J. Duck, P.J. Stuckey, and M. Sulzmann. Observable confluence for constraint handling rules. In CHR06, pages 61-76, 2006.

10. T. Frühwirth. Theory and practice of constraint handling rules. J. Log. Program., 37(1-3):95-138, 1998.

11. T. Frühwirth. Proving termination of constraint solver programs. In New Trends in Constraints, pages 298-317. Springer-Verlag, 2000.

12. T. Frühwirth and P. Brisset. Optimal placement of base stations in wireless indoor telecommunication. In CP98, pages 476-480. Springer-Verlag, 1998.

13. T. Schrijvers. Analyses, optimizations and extensions of constraint handling rules: Ph.d. summary. In ICLP05, pages 435-436, 2005.

14. T. Schrijvers and T. Frühwirth. Optimal union-find in constraint handling rules. TPLP, 6(1-2):213-224, 2006.

15. J. Sneyers, T. Schrijvers, and Demoen B. The computational power and complexity of constraint handling rules. In CHR05, pages 3-17, 2005.

16. D. Voets, P. Pilozzi, and D. De Schreye. A new approach to termination analysis of constraint handling rules. In CHRO\%, 2007. 